ŞUBĂ A., VACARAŞ O.

\title{
CENTER PROBLEM FOR CUBIC DIFFERENTIAL SYSTEMS WITH THE LINE AT INFINITY AND AN AFFINE REAL INVARIANT STRAIGHT LINE OF TOTAL MULTIPLICITY FOUR
}

\begin{abstract}
In this article, we show that a non-degenerate monodromic critical point of differential systems with the line at infinity and an affine real invariant straight line of total multiplicity four is a center type if and only if the first four Lyapunov quantities vanish.

Key words and phrases: Cubic differential system, center problem, invariant straight line.
\end{abstract}

Institute of Mathematics and Computer Science "V. Andrunachievici";

Tiraspol State University

Chişinău, Moldova (Şubă A., Vacaraş O.)

e-mail: alexandru.suba@math.md (Şubă A.), vacarasolga@yahoo.com (VacaraşO.)

\section{Introduction and statement of main results}

We consider the real cubic system of differential equations

$$
\left\{\begin{aligned}
\dot{x}= & y+a x^{2}+c x y+f y^{2}+k x^{3}+m x^{2} y+p x y^{2}+r y^{3} \equiv p(x, y), \\
\dot{y}= & -\left(x+g x^{2}+d x y+b y^{2}+s x^{3}+q x^{2} y+n x y^{2}+l y^{3}\right) \equiv q(x, y), \\
& \quad \operatorname{gcd}(p, q)=1
\end{aligned}\right.
$$

and the homogeneous system associated to the system (1)

$$
\left\{\begin{array}{l}
\dot{x}=y z^{2}+\left(a x^{2}+c x y+f y^{2}\right) z+k x^{3}+m x^{2} y+p x y^{2}+r y^{3} \equiv P(x, y, z), \\
\dot{y}=-\left(x z^{2}+\left(g x^{2}+d x y+b y^{2}\right) z+s x^{3}+q x^{2} y+n x y^{2}+l y^{3}\right) \equiv Q(x, y, z) .
\end{array}\right.
$$

Denote $\mathbb{X}=p(x, y) \frac{\partial}{\partial x}+q(x, y) \frac{\partial}{\partial y}$ and $\mathbb{X}_{\infty}=P(x, y, z) \frac{\partial}{\partial x}+Q(x, y, z) \frac{\partial}{\partial y}$.

An algebraic curve $f(x, y)=0, f \in \mathbb{C}[x, y]$ (a function $f=\exp (g / h), g, h \in \mathbb{C}[x, y]$ ) is called an invariant algebraic curve (exponential factor) of the system (1) if there exists a polynomial $K_{f} \in \mathbb{C}[x, y], \operatorname{deg}(K) \leq n-1$, such that the identity $\mathbb{X}(f) \equiv f(x, y) K_{f}(x, y)$, $(x, y) \in \mathbb{R}^{2} \quad\left((x, y) \in \mathbb{R}^{2} \backslash\left\{(x, y) \in \mathbb{R}^{2} \mid h(x, y)=0\right\}\right)$ holds. In particular, a straight line $l \equiv \alpha x+\beta y+\gamma=0, \alpha, \beta, \gamma \in \mathbb{C}$ is invariant for (1) if there exists a polynomial $K_{l} \in \mathbb{C}[x, y]$ such that the identity $\alpha P(x, y)+\beta Q(x, y) \equiv(\alpha x+\beta y+\gamma) K_{l}(x, y), \quad(x, y) \in \mathbb{R}^{2}$, holds. 
The invariant straight line $\alpha x+\beta y+\gamma=0$ (respectively, the line at infinity $z=0$ ) has the multiplicity $\nu$ (respectively, $\nu+1$ ) if $\nu$ is the greatest positive integer such that $(\alpha x+\beta y+\gamma)^{\nu}$ (respectively, $z^{\nu}$ ) divides $\mathbb{E}=p \cdot \mathbb{X}(q)-q \cdot \mathbb{X}(p)$ (respectively, $\mathbb{E}_{\infty}=P \cdot \mathbb{X}_{\infty}(Q)-Q \cdot \mathbb{X}_{\infty}(P)$ ) [5]. Denote by $m\left(l_{1}\right)\left(m\left(l_{\infty}\right)\right)$ the multiplicity of an affine invariant straight line $l_{1}$ (of the line at infinity $\left.l_{\infty}\right)$.

The cubic systems (1) with invariant straight lines were investigated in the works $[2,3$, $4,10,11,13,14,15,16,26,27,28,29,30]$,

The critical point $(0,0)$ of system $(1)$ is either a focus or a center, i.e. is monodromic. The problem of distinguishing between a center and a focus is called the center problem.

A critical point $(0,0)$ is a center for $(1)$ if and only if in a neighborhood of $(0,0)$ the system has a nonconstant analytic first integral $F(x, y)$ (an analytic integrating factor of the form $\left.\mu(x, y)=1+\sum \mu_{j}(x, y)\right)[12]([1])$.

It is known there exists a formal power series $F(x, y)=x^{2}+y^{2}+\sum_{j \geq 3} F_{j}(x, y)$ such that the rate of change of $F(x, y)$ along trajectories of (1) is a linear combination of polynomials $\left\{\left(x^{2}+y^{2}\right)^{j}\right\}_{j=2}^{\infty}$, i.e. $\frac{d F}{d t}=\sum_{j=2}^{\infty} L_{j-1}\left(x^{2}+y^{2}\right)^{j}$. The quantities $L_{j}, j=\overline{1, \infty}$, are polynomials with respect to the coefficients of system (1) called to be the Lyapunov quantities. The origin $(0,0)$ is a center for (1) if and only if $L_{j}=0, j=\overline{1, \infty}$. By the Hilbert basis theorem there is a natural number $N$ such that the infinite polynomial system $\left\{L_{j}=0, j=\overline{1, \infty}\right\}$ is equivalent to the finite system $\left\{L_{j}=0, j=\overline{1, N}\right\}$. We denote by $N_{0}$ the smallest such $\mathrm{N}$.

The center problem is completely solved for quadratic systems $(k=l=m=n=p=q=$ $r=s=0 ; N_{0}=3$ ) [9] and for symmetric cubic systems $\left(a=b=c=d=f=g=0 ; N_{0}=5\right)$ [20]. For other polynomial differential systems the necessary and sufficient conditions for the monodromic critical point to be a center were obtained in some particular cases (see, for example, $[6,17])$.

The problem of coexistence in cubic systems of distinct invariant straight lines and critical points of center type was studied in $[7,8,21,22,23,24,25]$. In these works it was proved that if the cubic system (1) has

- four non-homogeneous invariant straight lines, i.e. the lines of the form $1+\alpha_{j} x+\beta_{j} y=$ $0, j=1,2,3,4$, then $N_{0}=1[7] ;$

- two non-homogeneous and two homogeneous $(y \pm i x=0)$ invariant straight lines, then $N_{0}=2[8]$

- three invariant straight lines in generic position, then $N_{0}=3[25]$;

- a bundle of three invariant straight lines or three invariant straight lines two of which are parallel, then $N_{0}=5[21,24]$;

- two homogeneous and one non-homogeneous invariant straight lines, then $N_{0}=7$ [23].

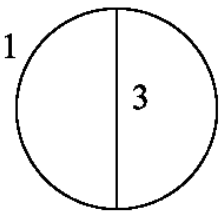

a)

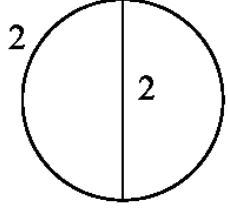

b)

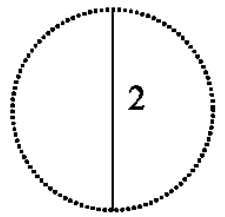

c)

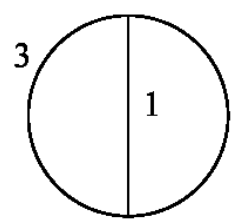

d)

Fig. 1.1 
In this article we investigate the center problem for cubic differential systems with the line at infinity and an affine real invariant straight line of total multiplicity four (see Fig. 1.1). Our main result is the following one:

Main Theorem. Each cubic system (1) with an affine real invariant straight line $l_{1}$ and the line at infinity $l_{\infty}$ of total multiplicity at least four: $m\left(l_{1}\right)+m\left(l_{\infty}\right) \geq 4$ has at critical point $(0,0)$ a center if and only if the first four Lyapunov quantities vanish $L_{1}=L_{2}=L_{3}=L_{4}=0$.

1 Solution of the CENTER PROBlem For CUBiC Systems With AN AFFine REAL INVARIANT STRAIGHT LINE OF MULTIPLICITY THREE

In this section we will solve for (1) the center problem in the case of configuration a) from Fig. 1.1.

\subsection{Classification of cubic systems with an affine real invariant straight line of multiplicity two or three}

Let the cubic system (1) have an affine real invariant straight line $l_{1}$. By a transformation of the form

$$
x \rightarrow \nu \cdot(x \cos \varphi+y \sin \varphi), y \rightarrow \nu \cdot(y \cos \varphi-x \sin \varphi), \nu \neq 0
$$

we can make $l_{1}$ to be described by the equation $x=1$. Then, $k=-a, m=-c-1, p=$ $-f, r=0$ and (1) is reduced to the system

$$
\left\{\begin{aligned}
\dot{x}= & (1-x)\left(y+a x^{2}+(c+1) x y+f y^{2}\right) \equiv p(x, y) \\
\dot{y}= & -\left(x+g x^{2}+d x y+b y^{2}+s x^{3}+q x^{2} y+n x y^{2}+l y^{3}\right) \equiv q(x, y) \\
& \operatorname{gcd}(P, Q)=1
\end{aligned}\right.
$$

For (2) we have

$$
\mathbb{E}=(x-1)\left(Y_{2}(y)+Y_{3}(y) \cdot(x-1)+\cdots+Y_{7}(y) \cdot(x-1)^{7}\right),
$$

where $Y_{j}(y), j=2, \ldots, 7$, are polynomials in $y$. The invariant line $x-1=0$ has multiplicity at least $j$ if the system of identities $\left\{Y_{2}(y) \equiv 0, \ldots, Y_{j}(y) \equiv 0\right\}$ holds. In particular, the line $x-1=0$ has multiplicity at least two (three) if $Y_{2}(y) \equiv 0 \quad\left(\left\{Y_{2}(y) \equiv 0, Y_{3}(y) \equiv 0\right\}\right)$. The polynomials $Y_{2}(y)$ and $Y_{3}(y)$ look as, respectively:

$$
\begin{aligned}
& Y_{2}(y)=\left(1+g+s+d y+q y+b y^{2}+n y^{2}+l y^{3}\right)\left(2+a^{2}+c-a d+2 g+c g-a q\right. \\
& \quad+2 s+c s+2(2 a-a b+a c+f+f g-a n+f s) y-((c+2)(b-2-c+n) \\
& \left.\quad-2 a f-d f+3 a l-f q) y^{2}+2(c+2)(f-l) y^{3}+f(f-l) y^{4}\right) \\
& Y_{3}(y)=a(g+2)(d+q)-\left(8-6 a^{2}+4 c+6 a d+4 g+2 c g+7 a q\right)(g+s+1) \\
& \quad+(7 c+13)(g+s+1)^{2}+(2 a((g+2)(b+n)+(3 a-2 d-3 q)(d+q)) \\
& \quad-(c+2)(g+2)(d+q)-(3 a+10 a b+d+8 f+4 f g+12 a n+q-(c+2) \\
& \left.\quad \cdot(9 a+5 d+6 q))(g+s+1)+12 f(g+s 1)^{2}\right) y+3(a((g+2) l+(2 a+d) \\
& \quad \cdot(b+n))-(a+4 a b-3 a(c+2)+2 f+f g+5 a n)(d+q)+\left(2+3 c+c^{2}\right.
\end{aligned}
$$




$$
\begin{aligned}
& +2 a f+4 d f-5 a l+5 f q)(g+s+1)) y^{2}+\left(15 a b-4 a b^{2}+9 a b c+6 d-3 b d\right. \\
& +9 c d-2 b c d+3 c^{2} d+3 f+2 b f+3 c f+6 a d f+2 d^{2} f+3 f g+4 b f g+3 c f g \\
& -3 l+6 a^{2} l-2 c l-12 a d l-5 g l-3 c g l+15 a n-12 a b n+9 a c n-5 d n-3 c d n \\
& +4 f n+6 f g n-8 a n^{2}+6 q-5 b q+9 c q-3 b c q+3 c^{2} q+6 a f q+6 d f q-16 a l q \\
& \left.-7 n q-4 c n q+4 f q^{2}+3 f s+6 b f s+3 c f s-7 l s-4 c l s+8 f n s\right) y^{3}+(f l-3 d f \\
& -b d f-3 a l+5 a b l+2 d l+2 f g l-3 f q-b f q+2 l q+3 f l s+(3 d f+9 a l-4 d l \\
& +3 f q-6 l q)(c+2)+(6 a f+2 d f-15 a l+3 f q)(b+n)+(c+2)(b+n)(3 c-b \\
& \left.-3 n+3)+(b+n)^{2}\right) y^{4}+3\left(2 a l(f-l)+l(b-1)(c+2)+l(c+2)^{2}\right. \\
& +(l-f)(b+n)+(f-2 l)(c+2)(b+n)) y^{5}+l((c+1)(3 f-2 l)-f n) y^{6} .
\end{aligned}
$$

Remark 1. If $1+g+s+d y+q y+b y^{2}+n y^{2}+l y^{3} \equiv 0$, then the system (2) is degenerate, i.e. $\operatorname{deg}(\operatorname{gcd}(p(x, y), q(x, y)))>0$.

Solving the identities $Y_{2}(y) \equiv 0$ and $\left\{Y_{2}(y) \equiv 0, Y_{3}(y) \equiv 0\right\}$, we obtain the following two Lemmas, respectively.

Lemma 1. The invariant straight line $x-1=0$ of the system (2) has multiplicity at least two if and only if one of the following four series of conditions holds:

$$
\begin{gathered}
a=f=0, c=-2 ; \\
a=f=l=0, n=2-b+c, s=-1-g, c \neq-2 ; \\
f=l=0, d=(a(a-q)+(c+2)(g+s+1)) / a, n=2-b+c ; \\
l=f, q=((c+2)(b-2-c+n)+f(a-d)) / f, \\
s=(-2 a+a b-a c-f-f g+a n) / f .
\end{gathered}
$$

Lemma 2. The invariant straight line $x-1=0$ of the system (2) has multiplicity at least three if and only if one of the following seven series of conditions holds:

$$
\begin{gathered}
a=d=f=l=q=0, b=1, n=c+1, s=-g-1, c \neq-2 ; \\
a=f=l=0, b=s=1, d=-q(c+3), g=-2, n=c+1, c \neq-2 ; \\
f=l=0, b=1, d=a(c+3) /(c+2), n=c+1, q=a(c+1) /(c+2), \\
s=\left(a^{2}-(c+2)(g+1)\right) /(c+2), a \neq 0 ; \\
f=l=0, b=s=1, d=(c+3)(a-q), g=a^{2}-a q-2, n=c+1, a \neq 0 ; \\
a=f=l=0, c=-2, n=-b, s=-g-1 ; \\
a=(1-b)(b-c-3) / f, q=((b-1)(2 c-b+5)-d f) / f, l=f, \\
g=\left((c-b+3)((b-1)(b-c-4)+d f)-2 f^{2}\right) / f^{2}, n=c+1, \\
s=\left((c-b+3)((c+3)(b-1)-d f)+f^{2}\right) / f^{2} ; \\
d=(b-1)(c+3) / f, g=-(a-a b+2 f) / f, l=f, n=c+1, \\
q=(a f-b+1) / f, s=1 .
\end{gathered}
$$




\subsection{Sufficient center conditions in the case $m\left(l_{1}\right) \geq 3$}

Remark 2. If $c+1 \neq 0$, then each of the systems $\{(2),(7)\}$ and $\{(2)$, (8) $\}$ has the parallel invariant straight lines: $x-1=0$ and $(c+1) x+1=0$.

\section{a) Darboux integrability.}

Let $f_{1}, \ldots, f_{r} \quad\left(f_{r+1}=\exp \left(g_{r+1} / h_{r+1}\right), \ldots, f_{s}=\exp \left(g_{s} / h_{s}\right)\right)$ be invariant algebraic curves (exponential factors) of (1) and let $K_{f_{j}}, j=\overline{1, s}$, be its cofactors. The system (1) is called Darboux integrable if (1) has a non-constant first integral (an integrating factor) of the form $F(x, y)=f_{1}^{\alpha_{1}} \cdots f_{s}^{\alpha_{s}}\left(\mu(x, y)=f_{1}^{\alpha_{1}} \cdots f_{s}^{\alpha_{s}}\right), \alpha_{j} \in \mathbb{C}, j=\overline{1, s}$ (on the theory of Darboux, presented in the context of planar polynomial differential systems on the affine plane, see [19]). Note that the constants $\alpha_{1}, \ldots, \alpha_{s}$ are not all equal to zero. If the system (1) is Darboux integrable, then the origin $(0,0)$ is a center.

Lemma 3. The following twelve sets of conditions are sufficient for the origin to be a center for system (2):

(i) $\quad a=d=f=l=q=0, b=1, n=1+c, s=-1-g, c \neq-2$;

(ii) $\quad f=l=n=q=0, b=1, c=-1, d=2 a, s=a^{2}-g-1$;

(iii) $\quad c=d=f=l=0, b=n=s=1, g=-2, q=a$;

(iv) $f=l=0, b=s=-n=1, c=-2, d=a-q, g=q(q-a)$, $q^{2}-a^{2}+2=0$

(v) $\quad a=f=l=0, b=1, c=-2, n=-1, q=d(g+1), s=-1-g$;

(vi) $\quad a=f=l=q=0, b=-c=-g=-n=2, s=1$;

(vii) $a=0, b=-n=s=1, c=g=-2, l=f, q=-d, d-f \neq 0$;

(viii) $\quad a=((1-b)(b-c-3)) / f, l=f, n=c+1$, $d=\left(2(b-1)(3-b+c)^{2}+(4-2 b+c) f^{2}\right) /((3-b+c) f)$, $g=\left((b-1)(b-c-3)(b-c-2)+(c-2 b+2) f^{2}\right) / f^{2}$, $q=\left((-1+b)^{2}(b-c-3)+(c-2 b+4) f^{2}\right) /((b-c-3) f)$, $s=(2 b-c-3)\left((b-1)(c-b+3)+f^{2}\right) / f^{2}, b-1 \neq 0$;

(ix) $\quad a=(c+2)^{2} /(4 f), b=(c+4) / 2, d=(c+2)(2 c+5) /(4 f), n=c+1$, $g=-(c+6) / 2, l=f, q=(c+1)(c+2) /(4 f), s=c /(2(1+c))$, $(c+1)(c+2)+4 f^{2}=0 ;$

(x) $\quad c=\left((b-1)\left(3 a-3 a b+5 f+2 a^{2} f-3 b f\right)+2 f^{3}(2-b)\right) / \beta$,

$d=(1-b)\left(3 a f-f^{2}+2 b f^{2}-\left(2 a^{2}-1\right)(b-1)\right) / \beta$,

$n=(2(b-1)(a+f)-f)\left(1-b+a f-f^{2}\right) / \beta, s=1$, $g=(a(b-1)-2 f) / f, l=f, q=(1-b+a f) / f, F_{1} F_{2}=0$,

where

$$
\begin{aligned}
& \beta=a(b-1)^{2}+(b-2)(b-1) f-f^{2}(a+f), \\
& F_{1}=a^{2} f-(b-1)^{2} f+a\left(b-b^{2}+f^{2}\right), \\
& F_{2}=(1-b)^{3}+2 a(b-1)^{2} f-2(b-1) f^{2}-2 f^{4}
\end{aligned}
$$

$$
a=\left(4 v^{3}\right) /\left(4 v^{2}-1\right), b=\left(1-8 v^{2}+8 v^{4}\right) /\left(1-4 v^{2}\right), c=4 v^{2} /\left(1-4 v^{2}\right) \text {, }
$$




$$
\begin{aligned}
& d=2 v\left(8 v^{2}-3\right) /\left(4 v^{2}-1\right), f=2 v\left(1-2 v^{2}\right) /\left(4 v^{2}-1\right) \\
& g=2\left(2 v^{2}-1\right)^{2} /\left(4 v^{2}-1\right), l=2 v\left(1-2 v^{2}\right) /\left(4 v^{2}-1\right) \\
& n=1 /\left(1-4 v^{2}\right), q=2 v\left(1-2 v^{2}\right) /\left(4 v^{2}-1\right), s=1
\end{aligned}
$$

(xii)

$$
\begin{aligned}
& a=\left(4 v^{3}\right) /\left(4 v^{2}-1\right), b=\left(1-8 v^{2}+8 v^{4}\right) /\left(1-4 v^{2}\right), \\
& c=2\left(4 v^{4}+v^{2}-1\right) /\left(1-4 v^{2}\right), d=2 v\left(1-10 v^{2}+8 v^{4}\right) /\left(1-4 v^{2}\right), \\
& f=2 v\left(2 v^{2}-1\right) /\left(1-4 v^{2}\right), g=2\left(2 v^{2}-1\right)^{2} /\left(4 v^{2}-1\right), \\
& l=2 v\left(2 v^{2}-1\right) /\left(1-4 v^{2}\right), n=\left(2 v^{2}-1\right)\left(1+4 v^{2}\right) /\left(1-4 v^{2}\right), \\
& q=2 v\left(2 v^{2}-1\right) /\left(1-4 v^{2}\right), s=1 .
\end{aligned}
$$

Proof. In all the cases (i)-(xii) the system (2) has in a neighborhood of origin $(0,0)$ an analytical integrating factor $\mu(x, y)$ :

in the case (i) $\mu(x, y)=1 /\left((x-1)^{3}(1+x+c x)\right)$;

in the case (ii) $\mu(x, y)=1 /(x-1)^{3}$;

in the case (iii) $\mu(x, y)=(x-1)^{-2} \exp \left[-y(y+2 a(x-1)) /(x-1)^{2}\right]$;

in the case (iv) $\mu(x, y)=\frac{1}{(x-1)^{2}} \exp \left[\frac{(q-3 a)(a-q+y)}{\left(2 q^{2}-2 a q+3\right)(x-1)}\right]$;

in the case $(\mathrm{v}) \mu(x, y)=(x-1)^{-3}(x-d y-1)^{-1}$;

in the case (vi) $\mu(x, y)=\frac{1}{(x-1)^{6}} \exp \left[\frac{d(-d+3 d x+6 y-6 x y)}{6(x-1)^{3}}\right]$;

in the case (vii) $\mu(x, y)=(x-1)^{\frac{4 d+d^{3}-4 f}{f-d}} \exp \left[\frac{\left.2 d(x-1)\left(d^{2}+d y-f y\right)-d^{2} f y^{2}\right)}{\left.2(d-f)(x-1)^{2}\right)}\right]$;

in the case (viii) $\mu(x, y)=l_{1}^{\alpha_{1}} l_{2}^{\alpha_{2}} l_{3}^{\alpha_{3}}$, where

$$
\begin{aligned}
l_{1}= & x-1, l_{2}=\exp [(f(b+f y-1)) /(x-1)], \\
l_{3}= & \exp \left[\left(\beta_{0}+\beta_{1} x+\beta_{2} y+\beta_{3} y^{2}\right) /\left((b-c-3)(x-1)^{2}\right),\right. \\
\beta_{0}= & 2(2 b-c-4) f^{2}-(b-1)(3 b-2 c-5)(b-c-3), \\
\beta_{1}= & 2\left((c-2 b+4) f^{2}+(b-1)(b-c-3)(2 b-c-3)\right), \\
\beta_{2}= & 2 f(b-1)(b-c-3), \beta_{3}=(b-c-3) f^{2}, \\
\alpha_{1}= & \left((c-2 b+4)^{3} f^{2}+(b-1)(b-c-3)\left(-52+92 b-48 b^{2}+8 b^{3}\right.\right. \\
& \left.\left.-44 c+48 b c-12 b^{2} c-12 c^{2}+6 b c^{2}-c^{3}\right)\right) /\left((b-1)(b-c-3)^{2}\right), \\
\alpha_{2}= & -(2 b-c-5)(2 b-c-4) /(f(b-c-3)), \\
\alpha_{3}= & -(2 b-c-4)^{2} /(2(b-1)(b-c-3)) ;
\end{aligned}
$$

in the case (ix) $\mu(x, y)=1 /\left(l_{1}^{3} \sqrt{l_{2}}\right)$, where $l_{1}=x-1$, $l_{2}=8(\beta-1)^{2}\left(\beta^{2}+1\right)-16(\beta-1)^{2}\left(1+\beta^{2}\right) x \pm 8\left(\beta^{4}-1\right) y+\left(\left(3-2 \beta+3 \beta^{2}\right) x \pm\left(1-\beta^{2}\right) y\right)^{2}$;

in the case $(\mathrm{x})$ $\mu(x, y)=(x-1)^{\alpha_{1}} \exp \left[(b-1+f y)(A+B x+C y) /\left(2 f \beta(b-1)(x-1)^{2}\right)\right]$,

where

$$
\begin{aligned}
& A=(1-b)\left((b-3)(b-1)^{2}-2 f(b-1)(2 a b+b f-a)+2 a f^{2}(2 a+f)\right), \\
& B=2(b-1)\left((b-2)(b-1)^{2}-a f\left(2 b^{2}-3 b+1\right)+f^{2}\left(1-b+2 a^{2}\right)\right), \\
& C=(b-1)^{3} f+2(b-1)^{2} f^{3}-2 a f^{4}, \alpha_{1}=-4+C /\left(f^{2} \beta\right) ;
\end{aligned}
$$

in the case $(\mathrm{xi}) \mu(x, y)=l_{2}^{\alpha_{2}} l_{3}^{\alpha_{3}} /(x-1)^{2}$, where 


$$
\begin{gathered}
l_{2}=\exp \left[\frac{2 v\left(1-2 v^{2}\right)(2 v+y)}{\left(4 v^{2}-1\right)(x-1)}\right] \\
l_{3}=\exp \left[\frac{4 v^{2}\left(2 v^{2}-1\right)^{2}(2 v+y)(-2 v+4 v x+y)}{\left.\left(4 v^{2}-1\right)^{2}(x-1)^{2}\right)}\right] \\
\alpha_{2}=\left(3-4 v^{2}\right) /\left(2 v^{2}-1\right), \alpha_{3}=\left(1-4 v^{2}\right) /\left(4 v^{2}\left(2 v^{2}-1\right)\right) ;
\end{gathered}
$$

in the case (xii) $\mu(x, y)=(x-1)^{\alpha_{1}} l_{2}^{\alpha_{2}} l_{3}^{\alpha_{3}}$, where $l_{2}, l_{3}$ are given in (15) and

$$
\alpha_{1}=8 v^{4}-2 v^{2}-4, \alpha_{2}=4 v^{2}-1, \alpha_{3}=\left(4 v^{2}-1\right)^{2} /\left(4\left(1-2 v^{2}\right)\right) .
$$

\section{b) The equation of nonlinear oscillations.}

Let $a=f=k=p=r=0$. Under these conditions the system (1) defines the following equation of nonlinear oscillations:

$$
P_{4}(x) y y^{\prime}=-x P_{0}(x)-3 x P_{1}(x) y-P_{2}(x) y^{2}-P_{3}(x) y^{3},
$$

where

$$
\begin{aligned}
& y^{\prime}=d y / d x, P_{0}(x)=1+g x+s x^{2}, P_{1}(x)=(d+q x) / 3, \\
& P_{2}(x)=b+n x, P_{3}(x)=l, P_{4}(x)=1+c x+m x^{2} .
\end{aligned}
$$

The substitution $y=\frac{P_{0}(x) Y}{1-P_{1}(Y)}$ [18] reduces the equation (16) to the form

$$
Q_{4}(x) Y Y^{\prime}=-x-Q_{2}(x) Y^{2}-Q_{3}(x) Y^{3},
$$

where

$$
\begin{aligned}
Q_{2}(x) \equiv & P_{0}(x) P_{2}(x)-3 x P_{1}^{2}(x)+P_{0}^{\prime}(x) P_{4}(x), \\
Q_{3}(x) \equiv & 2 x P_{1}^{3}(x)-P_{0}(x) P_{1}(x) P_{2}(x)+P_{0}^{2}(x) P_{3}(x) \\
& +P_{0}(x) P_{1}^{\prime}(x) P_{4}(x)-P_{0}^{\prime}(x) P_{1}(x) P_{4}(x), \\
Q_{4}(x) \equiv & P_{0}(x) P_{4}(x) .
\end{aligned}
$$

By [[1], Theorem 9.4], in the case $Q_{3}(x)=x^{2 j+1} \tilde{P}(x), \tilde{P}(0) \neq 0$, the origin is a center for differential equation (16) (system $\{(1), a=f=k=p=r=0\}$ ) if and only if the system of algebraic equations

$$
\begin{aligned}
& y^{4} R^{3}(x) Q_{3}^{5}(y)-x^{4} R^{3}(y) Q_{3}^{5}(x)=0, \\
& x Q(x) R^{2}(y)-y Q(y) R^{2}(x)=0,
\end{aligned}
$$

where

$$
\begin{aligned}
& R(x) \equiv Q_{4}(x)\left(Q_{3}(x)-x Q_{3}^{\prime}(x)\right)+3 x Q_{2}(x) Q_{3}(x), \\
& Q(x) \equiv Q_{4}(x)\left(R^{\prime}(x) Q_{3}(x)-3 R(x) Q_{3}^{\prime}(x)\right)+4 Q_{2}(x) Q_{3}(x) R(x)
\end{aligned}
$$

has in some neighborhood of $x=0$ a holomorphic solution $y=\varphi(x), \varphi(0)=0, \varphi^{\prime}(0)=-1$.

Lemma 4. The following set of conditions are sufficient for the origin to be a center for system (2):

$$
a=f=l=s=0, b=-n=1 / 3, c=-2, g=-1, q=-2 d / 3 .
$$


Proof. In conditions (18) the system (2) looks as

$$
\dot{x}=y(x-1)^{2}, \quad \dot{y}=-\left(3 x-3 x^{2}+3 d x y-2 d x^{2} y+y^{2}-x y^{2}\right) / 3 .
$$

For (19) we have:

$$
\begin{aligned}
& P_{0}(x)=1-x, P_{1}(x)=d(3-2 x) / 9, P_{2}(x)=(1-x) / 3, P_{3}(x)=0 \\
& P_{4}(x)=(x-1)^{2}
\end{aligned}
$$

and the system of equations (17) has the solution

$$
y=\frac{9-26 x+16 x^{2}+(2 x-3) \sqrt{9-8 x}}{16(x-1)^{2}} .
$$

\subsection{Solution of the center problem in the case $m\left(l_{1}\right) \geq 3$}

Theorem 1. The system (2) with invariant straight line $x-1=0$ of multiplicity three has a center at origin $(0,0)$ if and only if the first four Lyapunov quantities vanish.

Proof. To prove the theorem, we compute the first four Lyapunov quantities $L_{1}, L_{2}, L_{3}, L_{4}$ for each set of conditions (7)-(13) of Lemma 2. In the expressions for $L_{j}$ we neglect the denominators and non-zero constant factors.

The conditions (7) are the same as in Lemma 3, (i).

In the case (8) we have $c+2 \neq 0$. Therefore the vanishing of $L_{1}=(c+2) q$ gives $q=0$ and we are in the conditions $\{(\mathrm{i}), q=0\}$ of Lemma 3 .

In the case (9) $a \neq 0$ and the first Lyapunov quantity is $L_{1}=(1+c)(g+c+4)$. If $c=-1$, then Lemma 3, (ii). Let $a(c+1) \neq 0$ and $g=-c-4$. The second Lyapunov quantity looks as $L_{2}=a(c+1)(5+2 c)\left(a^{2}+(c+2)^{2}\right)$. If $c=-5 / 2$, then $L_{3}=a\left(1+4 a^{2}\right) \neq 0$. Therefore the origin is a focus.

In the case (10) the first Lyapunov quantity is $L_{1}=\left(a(a-q)^{2}+q-2 a\right) c+(a-q)\left(a^{2}-\right.$ $3 a q-2)$. Assume that

$$
a(a-q)^{2}+q-2 a \neq 0 .
$$

Then $L_{1}=0 \Rightarrow$

$$
c=(q-a)\left(a^{2}-3 a q-2\right) /\left(a(a-q)^{2}+q-2 a\right),
$$

and $L_{2}=f_{0} f_{2}$, where $f_{0}=a^{2} q(q-a)\left(a^{2}-q^{2}-2\right)\left((q-a)^{2}+1\right)$ and $f_{2}=\left(2-11 a^{2}+8 a q+\right.$ $\left.5 a^{2}(q-a)^{2}\right)$. If $q=0$ (respectively, $q=a ; a^{2}-q^{2}-2=0$ ), then Lemma $3,\left\{(\mathrm{ii}), g=a^{2}-2\right\}$ (respectively, (iii); (iv)). Let $f_{0} \neq 0$ and $f_{2}=0$. The Lyapunov quantities $L_{3}$ and $L_{4}$ have the form: $L_{3}=f_{0} f_{3}, L_{4}=f_{0} f_{4}$, where $f_{3}$ and $f_{4}$, reduced by $f_{2}$ in $a$, look as $f_{3}=162-$ $81 a^{2}+948 a q-420 a^{3} q+180 q^{2}+465 a^{2} q^{2}+745 a q^{3}+300 a^{3} q^{3}+300 a^{2} q^{4}$ and $f_{4}=-184941468+$ $92470734 a^{2}-831357432 a q+354031560 a^{3} q-282740220 q^{2}-324963900 a^{2} q^{2}-1781933580 a q^{3}+$ $816459750 a^{3} q^{3}-391131900 q^{4}-872231400 a^{2} q^{4}+269515275 a q^{5}-11098387875 a^{3} q^{5}+$ 
$2507899500 q^{6} 6-7129544250 a^{2} q^{6}+10021676125 a q^{7}-8736000000 a^{3} q^{7}+6329280000 a^{2} q^{8}$. The system in $a$ and $q$ : $\left\{f_{2}=0, f_{3}=0, f_{4}=0\right\}$ is not compatible. Suppose now that

$$
a(a-q)^{2}-2 a+q=0
$$

Then $L_{1}=(a-q)\left(-2+a^{2}-3 a q\right)$. Taking into account that $a \neq 0$ the system $\left\{a(a-q)^{2}-\right.$ $\left.2 a+q=0, L_{1}=0\right\}$ gives us $q=0, a^{2}-2=0$. Under these conditions the second and third Lyapunov quantities are $L_{2}=a(c+1)(c+2)(c+10), L_{3}=a(c+1)(c+2)(1394-1945 c+$ $\left.391 c^{2}+61 c^{3}\right)$. If $c=-1$, then Lemma $3,\left\{(\mathrm{ii}), a^{2}-2=0\right\}$ and if $c=-2$, then Lemma 3 , $\left\{(\right.$ iv $\left.), a^{2}-2=0\right\}$.

In the case (11) $L_{1}=d(b+g)-q=0 \Rightarrow q=d(b+g)$. Putting $q=d(b+g)$ in the second quantity we have $L_{2}=d(b+g+1)(b-1)(3 b+5 g+4)$. If $d(b+g+1)=0$, then the cubic system is degenerate: $\operatorname{gcd}(P, Q)=x-1$. Suppose $d(b+g+1) \neq 0$. If $b=1$, then Lemma 3, (v). Let $f_{0}=d(b-1)(b+g+1)^{2} \neq 0$ and $3 b+5 g+4=0 \Rightarrow g=-(3 b+4) / 5$. The third Lyapunov quantity looks as $L_{3}=f_{0}(b-2)(3 b-1)$. If $b=2$, then Lemma 3, (vi). If $b=1 / 3$, then Lemma 4 .

In the case (12) the first Lyapunov quantity is $L_{1}=f^{-3} g_{1} g_{2}$, where $g_{1}=d f(c-b+3)+$ $2(1-b)(c-b+3)^{2}+f^{2}(2 b-c-4)$ and $g_{2}=d f+(b-1)(b-c-4)+f^{2}$. Let $g_{1}=0$. If in $g_{1}$ the coefficient of $d$ is zero, i.e. $c=b-3$, then the equality $g_{1}=0$ yields $b=1$. In this subcase we are in condition (vii) of Lemma 3. Assume that $c-b+3 \neq 0$. Then $g_{1}=0 \Rightarrow d=\left(2(b-1)(c-b+3)^{2}+f^{2}(c-2 b+4)\right) /(f(c-b+3)) \Rightarrow$ Lemma 3 , (viii). Let now $g_{2}=0, g_{1} \neq 0$. From $g_{2}=0$ we express $d: \quad d=\left((b-1)(c-b+4)-f^{2}\right) / f$. The second and third Lyapunov quantities being cancelled by non-zero factors (as $f, g_{1},(b-1)^{2}+f^{2}$ ) have the form:

$$
\begin{aligned}
L_{2}= & (6 b-2 c-11)(b-c-3)-2 f^{2} \\
L_{3}= & \left((1-b)(2 b-3)-2 f^{2}\right)\left(553-1872 b+2359 b^{2}\right. \\
& \left.-1314 b^{3}+274 b^{4}+1175 f^{2}-1314 b f^{2}+548 b^{2} f^{2}+274 f^{4}\right)+\left((b-1)^{2}(2851\right. \\
& \left.-6240 b+4546 b^{2}-1096 b^{3}\right)+f^{2}\left(6625-13660 b+9636 b^{2}-2192 b^{3}-2898 f^{2}\right. \\
& \left.\left.-1096 b f^{2}\right)\right) \gamma+2\left((b-1)^{2}(193 b-241)+(193 b-157) f^{2}\right) \gamma^{2} \\
& +2\left((b-1)^{2}(548 b-629)+(548 b-1175) f^{2}\right) \gamma^{3}+548\left((b-1)^{2}+f^{2}\right) \gamma^{4},
\end{aligned}
$$

where $\gamma=c-2 b+4$. Solving the system $\left\{L_{2}=0, L_{3}=0\right\}$ we obtain $b=(c+4) / 2,(c+1)(c+$ $2)+4 f^{2}=0$. The equation $(c+1)(c+2)+4 f^{2}=0$ admits the following parametrization $c=\beta /\left(\beta^{2}+1\right)-3 / 2, f= \pm\left(\beta^{2}-1\right) /\left(4\left(\beta^{2}+1\right)\right)$. We are in the case (ix) of Lemma 3 .

In the case (13) we calculate the first four Lyapunov quantities and in their expressions we will cancel denominators and non-zero polynomial factors. The first quantity is $L_{1}=$ $\left(A_{c} c+B_{c}\right)$, where $A_{c}=a(b-1)^{2}+(b-2)(b-1) f-a f^{2}-f^{3}$ is the coefficient of $c$ in $L_{1}$, and $B_{c}=\left.L_{1}\right|_{c=0}=3 a(b-1)^{2}+(b-1)\left(3 b-2 a^{2}-5\right) f+2(b-2) f^{3}$. Two cases are possible: I) $A_{c} \neq 0$ and II) $A_{c}=B_{c}=0$.

I) $f A_{c} \neq 0$. We express $c$ from $L_{1}=0: c=-B_{c} / A_{c}$ and replacing in $L_{j}, j=2,3,4$, we obtain $L_{2}=F_{1} F_{2} F_{3}$ (see (14)), where $F_{3}=(a+f)^{2}(5 a+3 f)(b-1)^{2}-4 f(a+f)(2 a+f)(b-$ 1) $-f^{2}\left(3 a^{3}-2 a+11 a^{2} f+13 a f^{2}+5 f^{3}\right)$, and $L_{j}=F_{1} F_{2} F_{j+1}, j=3$, 4. The polynomials $F_{4}, F_{5}$ (in $\left.a, b, f\right)$ are very large and they are not given here. If $F_{1} F_{2}=0$, then Lemma 3 , 
(x). Suppose that $F_{1} F_{2} \neq 0$ and $F_{3}=0$. Taking into account that $f \neq 0$ the equality $F_{3}=0$ implies $(b-1)^{2}+a^{2} \neq 0$.

Later on, for short, instead of Resultant and PolynomialRemainder we will write Res and PRem respectively.

$\left.I_{1}\right)$ Let $b=1$, af $\neq 0$. Then $F_{3 b} \equiv-F_{3} / f^{2}=3 a^{3}-2 a+11 a^{2} f+13 a f^{2}+5 f^{3}$ and the remainder from dividing $\left(\left.F_{4}\right|_{b=1}\right) / f^{8}$ by $F_{3 b}$, treated as polynomials in $f$, is $-144 a^{2} F_{4 b} / 625$, where $F_{4 b}=250 a+242 a^{3}+12 a^{5}+125 f+430 a^{2} f+24 a^{4} f+200 a f^{2}+12 a^{3} f^{2}$. The Res $\left[F_{4 b}, F_{3 b}, f\right]$ $=390625 a\left(3 a^{2}+50\right) \neq 0$, i.e. the system $\left\{F_{3 b}=0, F_{4 b}=0\right\}$ has no solution.

$\left.I_{2}\right)$ Let $a=0, f(b-1) \neq 0$. In this case $F_{3 a} \equiv F_{3} / f^{3}=(b-1)(3 b-7)-5 f^{2}$ and $F_{4 a}=\operatorname{PRem}\left[\left(\left.F_{4}\right|_{a=0}\right) / f^{5}, F_{3 a}, f\right]=216(b-1)^{5}(2 b-3)^{4}(3 b-7) / 625$. If $F_{4 a}=0$, then $F_{3} \neq 0$.

We remark that if $a=-f$, then $F_{3} \neq 0$.

If $\gamma$ is a variable of polynomial $F$, then we denote by $L C(F, \gamma)$ the coefficient of greatest power of $\gamma$ in $F$. We have $L C\left(F_{3}, b\right)=(a+f)^{2}(5 a+3 f), L C\left(F_{4}, b\right)$ $=-2(a+f)^{4}\left(115 a^{3}+1330 a^{2} f+2491 a f^{2}+1032 f^{3}\right), L C\left(F_{5}\right)=-2(a+f)^{6}\left(36365 a^{5}-\right.$ $\left.14825 a^{4} f-1116816 a^{3} f^{2}-3290564 a^{2} f^{3}-3440437 a f^{4}-1109211 f^{5}\right)$. Because each of the systems $\left\{L C\left(F_{3}, b\right)=0, L C\left(F_{4}, b\right)=0\right\},\left\{L C\left(F_{3}, b\right)=0, L C\left(F_{5}, b\right)=0\right\}$ and $\left\{L C\left(F_{4}, b\right)=\right.$ $\left.0, L C\left(F_{5}, b\right)=0\right\}$ is not compatible, we have the right to calculate the following three resultants:

$$
\begin{aligned}
\operatorname{Res}\left[F_{3}, F_{4}, b\right]= & 5184 f^{16}(a+f)^{15}\left(4(a+f)^{2}+1\right)^{3}\left((a-f)^{2}(a+f)-2 a\right)^{3} G_{1}, \\
\operatorname{Res}\left[F_{3}, F_{5}, b\right]= & 20736 f^{28}(a+f)^{19}\left(4(a+f)^{2}+1\right)^{5}\left((a-f)^{2}(a+f)-2 a\right)^{5} G_{2}, \\
\operatorname{Res}\left[F_{4}, F_{5}, b\right]= & 80244904034304 f^{112}(a+f)^{33}\left(4(a+f)^{2}+1\right)^{15} \\
& \cdot\left((a-f)^{2}(a+f)-2 a\right)^{15} G_{3},
\end{aligned}
$$

where $G_{1}, G_{2}, G_{3}$ are polynomials in $a$ and $f$.

$\left.I_{3}\right)$ Let $a f(b-1)(a+f) F_{1} F_{2} \neq 0$ and $(a-f)^{2}(a+f)-2 a=0$. The transformation $a \rightarrow u+v, f \rightarrow u-v$ reduces this equality to $\left(4 v^{2}-1\right) u-v=0$. Express $u$ from the last equality: $u=v /\left(4 v^{2}-1\right)$ and substitute it in $F_{3}, F_{4}$ and $F_{5}$. We have $F_{3}=8 v^{3}(1-$ $\left.b-8 v^{2}+4 b v^{2}+8 v^{4}\right) F_{3 b v} /\left(4 v^{2}-1\right)^{5}, \quad F_{4}=32 v^{5}\left(1-b-8 v^{2}+4 b v^{2}+8 v^{4}\right)^{3} F_{4 b v} /\left(4 v^{2}-1\right)^{15}$, $F_{5}=128 v^{7}\left(1-b-8 v^{2}+4 b v^{2}+8 v^{4}\right)^{5} F_{5 b v} /\left(4 v^{2}-1\right)^{25}$, where $F_{3 b v}=\left(b\left(4 v^{2}+3\right)\left(4 v^{2}-1\right)+\right.$ $\left.32 v^{6}-40 v^{4}-12 v^{2}+7\right)$ and $F_{4 b v}, F_{5 b v}$ are polynomials in $b, v$.

If $v\left(1-b-8 v^{2}+4 b v^{2}+8 v^{4}\right)=0$, then $F_{1}=0$. The equation $F_{3 b v}=0$ yields $b=$ $\left(32 v^{6}-40 v^{4}-12 v^{2}+7\right) /\left(\left(4 v^{2}+3\right)\left(1-4 v^{2}\right)\right)$. In this condition $F_{4 b v}=-576 v^{2}\left(16 v^{4}-1\right)^{3}\left(2 v^{2}-\right.$ $1)^{5} F_{4 v} /\left(4 v^{2}+3\right)^{5}, F_{5 b v}=-2304 v^{2}\left(\left(16 v^{4}-1\right)^{5}\right)\left(\left(2 v^{2}-1\right)^{9}\right) F_{5 v} /\left(4 v^{2}+3\right)^{9}$, where $F_{4 v}=-30-$ $123 v^{2}+6 v^{4}+104 v^{6}-352 v^{8}+128 v^{10}, F_{5 v}=-137160-5742894 v^{2}-21786777 v^{4}+24074466 v^{6}+$ $94898504 v^{8}-206114048 v^{10}-194681088 v^{12}+591481856 v^{14}-312180736 v^{16}-597753856 v^{18}+$ $865468416 v^{20}-409862144 v^{22}+66584576 v^{24}$. The polynomial $v\left(16 v^{4}-1\right)\left(2 v^{2}-1\right)$ and the resultant $\operatorname{Res}\left[F_{4 v}, F_{5 v}, v\right]$ are not equal to zero. Therefore, the equality $(a-f)^{2}(a+f)-2 a=0$ does not give any new cases of center for system (2) in conditions (13).

$\left.I_{4}\right)$ Let now $a f(b-1)(a+f)\left((a-f)^{2}(a+f)-2 a\right) F_{1} F_{2} \neq 0$ and $G_{1}=G_{2}=G_{3}=0$. The leading coefficients $L C\left(G_{1}, a\right), L C\left(G_{2}, a\right)$ and $L C\left(G_{3}, a\right)$ are non-zero natural numbers. We calculate the resultants: $\operatorname{Res}\left[G_{1}, G_{2}, a\right]=f^{44}\left(8+285 f^{2}+2880 f^{4}\right) G_{12 f}, \operatorname{Res}\left[G_{1}, G_{3}, a\right]=$ $f^{86}\left(8+285 f^{2}+2880 f^{4}\right) G_{13 f}$, where $G_{12 f}, G_{13 f}$ are polynomials in $f$ and $\operatorname{Res}\left[G_{12 f}, G_{13 f}, f\right] \neq$ 0 . 
From subsections $\left.\left.I_{1}\right)-I_{4}\right)$ it follows that in the case of $A_{c} F_{1} F_{2} \neq 0$ the system $\{(2)$, (13) $\}$ has a focus at $(0,0)$.

II) Let now $A_{c}=B_{c}=0$. These equalities are equivalent with equalities $A_{c}=\tilde{B}_{c}=0$, where $\tilde{B}_{c}=\left(3 A_{c}-B_{c}\right) / f=\left(2 a^{2}-2 f^{2}-1\right) b+f^{2}-2 a^{2}-3 a f+1$. The system of equations $\left\{2 a^{2}-2 f^{2}-1=0, f^{2}-2 a^{2}-3 a f+1=0\right\}$ is not compatible. We express $b$ from $\tilde{B}_{c}=0$ : $b=\left(2 a^{2}+3 a f-f^{2}-1\right) /\left(2 a^{2}-2 f^{2}-1\right)$. Then $A_{c}=-f^{2}\left(1+4(a+f)^{2}\right)\left((a-f)^{2}(a+f)-\right.$ $\left.2 a) /\left(2 a^{2}-2 f^{2}-1\right)^{2}\right)=0 \Rightarrow(a-f)^{2}(a+f)-2 a=0$. As for $\left.I_{3}\right)$, via the transformation $a \rightarrow u+v, f \rightarrow u-v$ the equality $(a-f)^{2}(a+f)-2 a=0$ takes the form $\left(4 v^{2}-1\right) u-v=0$. We substitute $u=v /\left(4 v^{2}-1\right)$ in $L_{2}: L_{2}=v f_{1} f_{2} f_{3}$, where $f_{1}=\left(4 v^{2}+c\left(4 v^{2}-1\right)\right), f_{2}=$ $c\left(4 v^{2}-1\right)+2\left(4 v^{4}+v^{2}-1\right), f_{3}=24 v^{2}-2+c\left(4 v^{2}-1\right)$. If $v=0$, then $u=0$, but $\mathrm{u}=\mathrm{v}$ $=0$ implies $f=0$ which cannot be in our case. If $f_{1}=0 \quad\left(f_{2}=0\right)$, then Lemma 3 , (xi) (Lemma 3, (xii)). Finally, we will examine the subcase $f_{3}=0$. The equality $f_{3}=0$ yields $c=\left(24 v^{2}-2\right) /\left(1-4 v^{2}\right) \Rightarrow L_{3}=4608 v^{5} f_{1} f_{2} g_{1} /\left(4 v^{2}-1\right)^{5}, L_{4}=v^{5} f_{1} f_{2} g_{2} /\left(30\left(1-4 v^{4}\right)^{7}\right)$, where $g_{1}=7+34 v^{2}-8 v^{4}, g_{2}=6013-136338 v^{2}+900152 v^{4}+8431680 v^{6}-2754304 v^{8}+$ $1340928 v^{10}-260096 v^{12}$. The system $\left\{g_{1}=0, g_{2}=0\right\}$ has no solution.

Example 1. We consider the system (2). If

$$
\begin{aligned}
& a=\sqrt{(3 \sqrt{5}-5) / 30}, b=s=1, c=(\sqrt{5}-5) / 2, d=\sqrt{3(5+3 \sqrt{5}) / 10} \\
& f=l=0, g=-(15+\sqrt{5}) / 10, n=(\sqrt{5}-3) / 2, q=-\sqrt{(27 \sqrt{5}-35) / 30}
\end{aligned}
$$

then $m\left(l_{1}\right)=3$ and $L_{1}=L_{2}=L_{3}=0, L_{4} \neq 0$.

Denote $C_{3}=s x^{4}+(k+q) x^{3} y+(m+n) x^{2} y^{2}+(l+p) x y^{3}+r y^{4}$. If $C_{3} \equiv 0$, then the system (1) has degenerate infinity, i.e. the infinity consists only of singular points.

2 Solution of the Center problem for Cubic systems With AN AfFine REAL INVARIANT STRAIGHT LINE OF MULTIPLICITY TWO AND $m\left(l_{\infty}\right) \geq 2$ OR $C_{3} \equiv 0$

In this section we will solve for (1) the center problem in the cases of configurations b) and c) from Fig. 1.1, i.e. in the cases $m\left(l_{1}\right) \geq 2$ and $m\left(l_{\infty}\right) \geq 2$ or $C_{3} \equiv 0$.

We consider $l_{1}=x-1$, then system (1) looks as (2). For (2) we have $C_{3}=x \tilde{C}_{3}$, where $\tilde{C}_{3}=s x^{3}+(q-a) x^{2} y+(n-c-1) x y^{2}+(l-f) y^{3}$.

\subsection{Classification of cubic systems (2) in the case $m\left(l_{1}\right) \geq 2$ and $m\left(l_{\infty}\right) \geq 2$ or $C_{3} \equiv 0$}

For (2) we have

$$
\mathbb{E}_{\infty}=(x-z) \cdot\left(\tilde{C}_{3} \cdot H_{2}(x, y)+H_{3}(x, y) z+\cdots+H_{7}(x, y) z^{5}\right),
$$

where $H_{j}(x, y), j=2, \ldots, 7$, are polynomials in $x$ and $y$. 
The line at infinity has multiplicity at least $j$ if the system of identities $\left\{H_{2}(x, y) \equiv\right.$ $\left.0, \ldots, H_{j}(x, y) \equiv 0\right\}$ holds. In particular, the line at infinity has multiplicity at least two if $H_{2}(x, y) \equiv 0$. The polynomial $H_{2}(x, y)$ looks as:

$H_{2}(x, y)=(a q-s-c s) x^{4}+2(a n-f s) x^{3} y+(3 a l+n+c n-f q) x^{2} y^{2}+2(c+1) l x y^{3}+f l y^{4}$.

Solving the identities $H_{2}(x, y) \equiv 0$ and $C_{3} \equiv 0$ in each of the sets of conditions (3)-(6) we obtain the following two Lemmas, respectively.

Lemma 5. Both the line at infinity and the invariant straight line $x-1=0$ of the system (2) have multiplicity at least two if and only if one of the following four series of conditions holds:

$$
\begin{gathered}
a=f=l=n=s=0, c=-2 ; \\
a=f=l=0, c=-1, n=1-b, s=-1-g ; \\
a=f=l=n=s=0, b=c+2, g=-1, c+2 \neq 0 ; \\
f=l=n=0, b=c+2, d=\left((c+2)(g+1)+s+a^{2}\right) / a, q=s(c+1) / a .
\end{gathered}
$$

Lemma 6. Let the invariant straight line $x-1=0$ of the system (2) have multiplicity at least two. Then for this system the infinity is degenerate if and only if one of the following four series of conditions holds:

$$
\begin{gathered}
a=f=l=q=s=0, c=-2, n=-1 ; \\
a=f=l=q=s=0, b=1, g=-1, n=c+1, c+2 \neq 0 ; \\
b=1, d=(c+2)(g+1) / a, f=l=s=0, n=c+1, q=a ; \\
d=(b-1)(c+2) / f, g=(a b-a-f) / f, l=f, n=c+1, q=a, s=0 .
\end{gathered}
$$

\subsection{Sufficient center conditions in the cases $m\left(l_{1}\right) \geq 2$ and $m\left(l_{\infty}\right) \geq 2$ or $C_{3} \equiv 0$}

\section{a) Darboux integrability.}

Lemma 7. The following twelve sets of conditions are sufficient for system (2) to have at origin a center:

(i) $\quad a=b=f=l=n=s=0, c=-2, q=d g$;

(ii) $\quad a=d=f=l=n=q=s=0, c=-2$;

(iii) $\quad a=f=l=n=0, b=-c=1, q=d(g+1), s=-1-g$;

(iv) $\quad a=d=f=l=q=0, c=-1, n=1-b, s=-1-g$;

(v) $\quad a=f=l=n=s=0, b=c+2, g=-1, q=d(c+1), c+2 \neq 0$;

(vi) $\quad b=c+2, d=\left(2 a^{2}-(c+1)(c+2)\right) / a, f=l=n=0$, $q=(c+1)\left(a^{2}-(c+2)(c+g+2)\right) / a, s=a^{2}-(c+2)(c+g+2)$;

(vii) $\quad a=d=f=l=q=s=0, c=-2, n=-1$;

(viii) $\quad a=f=l=q=s=0, c=-2, g=-b, n=-1$; 
(ix) $\quad a=f=l=q=s=0, b=1, g=-1, n=c+1, c+2 \neq 0$

(x) $\quad b=1, d=(c+2)(g+1) / a, f=l=s=0, n=c+1, q=a$, $a^{2}(c+2 g+4)-(c+2)(g+1)^{2}=0$; $d=(b-1)(c+2) / f, g=(a b-a-f) / f, l=f, n=c+1, q=a$, $s=0,2\left(f^{2}-(b-1)^{2}+(b-1)(a-f) f\right)+c\left(f^{2}-(b-1)^{2}\right)=0 ;$

(xii) $\quad a=q=-f, d=(b-1)(c+2) / f, g=-b, l=f, n=c+1, s=0$.

Proof. In all the cases (i)-(xii) the system (2) has in a neighborhood of origin $(0,0)$ an analytical integrating factor $\mu(x, y)$ :

in the case (i) $\mu(x, y)=(x-1)^{-2}(1+d y)^{-1}$;

in the case (ii) $\mu(x, y)=(x-1)^{-2} \exp [2 b x /(1-x)]$;

in the case (iii) $\mu(x, y)=(x-1)^{-3-d^{2}(3+2 g)} \exp \left[\frac{d(d(g+2)+y)}{x-1}-d^{2}(g+1) x\right]$;

in the case (iv) $\mu(x, y)=(x-1)^{-3} \exp [2(b-1) x]$;

in the case $(\mathrm{v}) \mu(x, y)=\frac{(c+1) x+1}{(x-1)^{3+d^{2}(2 c+3)}} \exp \left[\frac{d(c+2)(d+y)}{x-1}+d(c+1)(y-d x)\right]$;

in the case (vi) $\mu(x, y)=\frac{1}{(x-1)^{2}((c+2)(a x+y+c y)-a)}$;

in the case (vii) $\mu(x, y)=(x-1)^{-4} \exp [2(1-b) /(x-1)]$;

in the case (viii) $\mu(x, y)=1 /((x-1) \Psi)$, where $\Psi=(b x-1)^{2}-d(b x-1) y-(b-1)^{2} y^{2}$ (note that $\Psi$ is reducible in $\mathbb{R}[x, y]$, i.e. $\Psi=l_{2} l_{3}$, where $l_{2}$ and $l_{3}$ are straight lines with real coefficients);

in the case (ix) $\mu(x, y)=(x-1)^{-3-\frac{d^{2}}{(c+2)^{2}}}(1+x+c x)^{-1+\frac{d^{2}}{(c+2)^{2}}} \exp \left[\frac{d(d+2 y+c y)}{(c+2)(x-1)}\right]$;

in the case $(\mathrm{x}) \mu(x, y)=\frac{1}{(x-1)^{4}} \exp \left[\frac{\left((c+2)(g+1)-2 a^{2}\right)(1+g+a y)}{a^{2}(x-1)}\right]$;

in the case $(\mathrm{xi}) \mu(x, y)=\frac{1}{(x-1)^{4}} \exp \left[\frac{((b-1)(c+2)-2 a f)(b-1+f y)}{f^{2}(x-1)}\right]$;

in the case (xii) $\mu(x, y)=1 /\left((x-1)^{2} \Phi\right)$, where $\Phi=f-f(2 b-c-2) x+\left(2 f^{2}+(b-1)(c+\right.$ $2)) y+f\left(b(b-c-2)-f^{2}\right) x^{2}+\left(c f^{2}-(b-1)(c+2)(b-c-2)\right) x y+f\left(f^{2}-(b-1)(b-c-3)\right) y^{2}$ (note that $\Phi=l_{1} l_{2}$, where $l_{2}$ and $l_{3}$ are straight lines with real coefficients).

b) Reversibility.

The system (1) (in particular, the system (2)) has a center at $O(0,0)$ if there exists a diffeomorphism

$$
\Phi: U \rightarrow V, \Phi=\{x=\varphi(X, Y), y=\psi(X, Y)\}, \Phi(0,0)=(0,0)
$$

which brings system (1.1) to a system with the axis of symmetry. In particular, if $\varphi(X, Y)$ and $\psi(X, Y)$ are rational functions, then we say that (1) is rationally reversible ([31]).

In this subsection we consider the diffeomorphism of the form

$$
\Phi=\left\{x=\frac{a_{1} X+b_{1} Y}{a_{3} X+b_{3} Y-1}, y=\frac{a_{2} X+b_{2} Y}{a_{3} X+b_{3} Y-1}\right\}
$$

$a_{1} b_{2}-a_{2} b_{1} \neq 0$. Applying the transformation (30) to (1) we obtain a system of the form $\dot{X}=$ $R(X, Y) / L(X, Y), \dot{Y}=S(X, Y) / L(X, Y)$ which is equivalent in a neighborhood of $O(0,0)$ with the system $\dot{X}=R(X, Y), \dot{Y}=S(X, Y)$. If $R(X, Y)=Y+M\left(X^{2}, Y\right), S(X, Y)=$ $-X\left(1+N\left(X^{2}, Y\right)\right)$, i.e.

$$
\dot{X}=Y+M\left(X^{2}, Y\right), \quad \dot{Y}=-X\left(1+N\left(X^{2}, Y\right)\right),
$$


then for (31) $X=0$ is an axis of symmetry and therefore the system (1) has a center at $O(0,0)$.

Lemma 8. The following set of conditions are sufficient for the origin to be a center for system (2):

$$
c=-3 b=-3 / 2, d=\left(a^{2}+s\right) / a, f=g+1=l=n=0, q=-s /(2 a) .
$$

Proof. In conditions (32) the system (2) looks as

$$
\begin{aligned}
& \dot{x}=(1-x)\left(2 y+2 a x^{2}-x y\right) / 2, \\
& \dot{y}=-\left(2 a x-2 a x^{2}+2\left(a^{2}+s\right) x y+a y^{2}+2 a s x^{3}-s x^{2} y\right) /(2 a) .
\end{aligned}
$$

In a neighborhood of $O(x, y)$ the transformation

$$
\Phi=\left\{x=\frac{2 X}{X+2}, y=\frac{2 Y}{X+2}\right\}
$$

reduces (33) to a system of the form (31):

$$
\begin{aligned}
& \dot{X}=Y+X^{2}\left(4 a-Y-a X^{2}\right) / 4, \\
& \dot{Y}=-X\left(4 a+4\left(a^{2}+s\right) Y+a(4 s-1) X^{2}+2 a Y^{2}+a^{2} X^{2} Y\right) /(4 a) .
\end{aligned}
$$

\subsection{Solution of the center problem in the cases $m\left(l_{1}\right) \geq 2$ and $m\left(l_{\infty}\right) \geq 2$ or $C_{3} \equiv 0$}

Theorem 2. The system (2) with the line at infinity and the invariant straight line $x-1=0$ both of multiplicity two has a center at origin $(0,0)$ if and only if the first four Lyapunov quantities vanish.

Proof. Assume one of the sets of conditions (22)-(25) of Lemma 5 holds. In the case (22) the first Lyapunov quantity looks as $L_{1}=d(b+g)-q$. If $q=d(b+g)$, then $L_{2}=b d G_{2}, L_{3}=$ $b d G_{3}, L_{4}=b d G_{4}$, where $G_{2}, G_{3}, G_{4}$ are polynomial in $b, d, g$. The remainders after dividing $G_{3}$ and $G_{4}$ by $G_{2}=1+6 b+3 b^{2}+6 g+8 b g+5 g^{2}$ are, respectively, $G_{3 g} \equiv \operatorname{PRem}\left[G_{3}, G_{2}, g\right]=$ $2+4 b+6 b^{2}+14 b^{3}-6 b^{4}+2 g+3 b g+14 b^{2} g-6 b^{3} g$ and $G_{4 g} \equiv P \operatorname{Rem}\left[G_{4}, G_{2}, g\right]=2(11079+$ $\left.40677 b+67806 b^{2}+82222 b^{3}+143414 b^{4}+119560 b^{5}-60168 b^{6}\right)+\left(22158+70275 b+103500 b^{2}+\right.$ $\left.226660 b^{3}+239120 b^{4}-120336 b^{5}\right) g+3175 d^{2} G_{3 g}$. If $b=0 \quad(d=0)$, then Lemma 7 , (i) (Lemma 7 , (ii)) and if $b d \neq 0$, then the system $G_{2}=G_{3 g}=G_{4 g}=0$ has no solution.

Case (23). We have $L_{1}=d(b+g)-q$ and $L_{1}=0 \Rightarrow q=d(b+g) \Rightarrow L_{j}=d(b-1) F_{j}, j=$ $2,3,4$. If $b=1 \quad(d=0)$, then Lemma 7 , (iii) (Lemma 7, (iv)). Let $d(b-1) \neq 0$. The system in $b, d, g:\left\{F_{2}=F_{3 g}=F_{4 g}=0, b-1 \neq 0\right\}$, where $F_{2}=2+4 b+3 b^{2}+6 g+8 b g+5 g^{2}, F_{3 g} \equiv$ $P \operatorname{Rem}\left[F_{3}, F_{2}, g\right]=2\left(3+4 b-10 b^{2}+b^{3}+3 b^{4}\right)+\left(13-13 b-4 b^{2}+6 b^{3}\right) g, F_{4 g} \equiv P \operatorname{Rem}\left[F_{4}, F_{2}, g\right]=$ 
$2\left(887-3052 b+98954 b^{2}+20788 b^{3}-216554 b^{4}+51688 b^{5}+60168 b^{6}\right)+\left(4977+4645 b+268740 b^{2}-\right.$ $\left.355980 b^{3}-16960 b^{4}+120336 b^{5}\right) g+3175 F_{3 g} d^{2}$, is incompatible.

In the case (24) the first Lyapunov quantity is $L_{1}=d(c+1)-q$. If $q=d(c+1)$, then Lemma $7,(\mathrm{v})$.

Under conditions (25) the first two Lyapunov quantities look as: $L_{1}=f_{1} f_{2}, L_{2}=f_{1} f_{3}$, where $f_{1}=\left(a^{2}-(c+2)(c+g+2)-s\right), f_{2}=g+1$ and $f_{3}=8 a^{4}(3+2 c)-4\left(a^{2}\left(33+5 a^{2}+\right.\right.$ $\left.\left.30 c+7 c^{2}-4 s\right)+s^{2}\right) f_{2}-2\left(8 a^{2}+a^{2} c+8 s+4 c s\right) f_{2}^{2}-\left(5 a^{2}+4(2+c)^{2}\right) f_{2}^{3}$. If $f_{1}=0$, then Lemma $7,(\mathrm{vi})$, and if $f_{2}=f_{3}=0$, i.e. $g=-1, c=-3 / 2$, then Lemma 8 .

Example 2. We consider the system (2). If $a=f=l=n=s=0, b=(\sqrt{10}-$ $1) / 3, c=-2, g=-2 \sqrt{10} / 5, q=-(\sqrt{10}+5) d / 15$, then $m\left(l_{1}\right)=2, m\left(l_{\infty}\right)=2, C_{3} \neq 0$ and $L_{1}=L_{2}=L_{3}=0, L_{4} \neq 0$.

Theorem 3. The system (2) with degenerate infinity $\left(C_{3} \equiv 0\right)$ and the invariant straight line $x-1=0$ of multiplicity two has a center at origin $(0,0)$ if and only if the first Lyapunov quantity vanishes.

Proof. In the case (26) the first Lyapunov quantity looks as $L_{1}=d(g+b)$. If $d=0$, then Lemma 7, (vii), and if $g=-b$, then Lemma 7, (viii).

The conditions (27) are the same as in Lemma 7, (ix).

In the case (28) if $L_{1}=0$, i.e. $a^{2}(4+c+2 g)-(2+c)(1+g)^{2}=0$, then Lemma $7,(\mathrm{x})$.

In the case $(29)$ the quantity $L_{1}$ has the form $L_{1}=(a+f) g_{1}$, where $g_{1}=2\left(f^{2}-(b-\right.$ $\left.1)^{2}+(b-1)(a-f) f\right)+c\left(f^{2}-(b-1)^{2}\right)$. If $g_{1}=0$, then Lemma 7, (xi) and if $a+f=0$, then Lemma 7, (xii).

\section{Solution of the CEnTER PROBlem For CUbiC Systems With AN AFFine}

REAL INVARIANT STRAIGHT LINE AND THE LINE AT INFINITY OF MULTIPLICITY AT LEAST THREE

In this section we will solve for (1) the center problem in the case of configuration d) from Fig. 1.1.

Without loss of generality we consider that the real invariant straight line is described by equation $x-1=0$, i.e. the system (1) has the form (2).

Theorem 4. The system (2) with the line at infinity of multiplicity at least three has a center at origin $(0,0)$ if and only if the first four Lyapunov quantities vanish.

Proof. The transformation $X=x /(x-1), Y=y /(x-1)$ reduces the system (2) to the system

$$
\begin{aligned}
\dot{X}=( & X-1)\left(-Y+a X^{2}+(c+2) X Y+f Y^{2}\right), \\
\dot{Y}=- & \left(X-(g+2) X^{2}-d X Y+(1-b) Y^{2}+(g+s+1) X^{3}\right. \\
& \left.+(d+q-a) X^{2} Y+(b+n-c-2) X Y^{2}+(l-f) Y^{3}\right) .
\end{aligned}
$$

The line at infinity for (34) has multiplicity at least three if and only if one of the sets of conditions (7)-(13) holds. Moreover, the system (34) with the line at infinity of multiplicity 
at least three has at $(0,0)$ a center if and only if at least one of the series of conditions of Lemma 3 and Lemma 4 is satisfied.

Under the conditions (21) the line at infinity has multiplicity three for system (34) and $L_{1}=L_{2}=L_{3}=0, L_{4} \neq 0$.

The Main Theorem results from Sections 1-3.

\section{REFERENCES}

[1] Amelkin V.V., Lukashevich N.A., Sadovskii A.P. Non-linear oscillations in the systems of second order. Belorusian University Pres. Minsk. 1982 (in Russian).

[2] Bujac C., Schlomiuk D. and Vulpe N. Configurations of invariant straight lines of the type $(2,2,1,1)$ for a family of cubic systems. In: Proc. of the Fifth Conf. of Math. Soc. of the Rep. of Moldova., Chişinău, 2019, 24-27.

[3] Bujac C., Vulpe N. Cubic systems with invariant straight lines of total multiplicity eight and with three distinct infinite singularities. Qual. Theory Dyn. Syst. 2015, 14 (1), 109-137.

[4] Bujac C., Vulpe N. Cubic systems with invariant lines of total multiplicity eight and with four distinct infinite singularities. Journal of Mathematical Analysis and Applications. 2015, 423 (2), 1025-1080.

[5] Christopher C., Llibre J., Pereira J.V. Multiplicity of invariant algebraic curves in polynomial vector fields. Pacific J. of Math. 2007, 229 (1), 63-117.

[6] Cozma D. Integrability of cubic systems with invariant straight lines and invariant conics. Ştiinţa, Chişinău, 2013.

[7] Cozma D., Şubă A. Partial integrals and the first focal value in the problem of centre. Nonlin. Diff. Equ. and Appl. 1995, 2, 21-34.

[8] Cozma D., Şubă A. The solution of the problem of center for cubic differential systems with four invariant straight lines. An. Ştiinţ. Univ. "Al. I. Cuza" (Iaşi). 1998, 44, suppl., 517-530.

[9] Dulac H. Détermination et intégration d'une certaine classe d'équations différentielles ayant pour point singulier un centre. Bull. Sciences Math. 1908, 32, 230-252.

[10] Kooij R. Cubic systems with four line invariants, including complex conjugated lines. Math. Proc. Camb. Phil. Soc. 1995, 118 (1), 7-19.

[11] Llibre J. and Vulpe N. Planar cubic polynomial differential systems with the maximum number of invariant straight lines. Rocky Mountain J. Math. 2006, 36 (4), 1301-1373.

[12] Lyapunov A.M. The general problem of the stability of motion. Gostekhizdat, Moscow, 1950 (in Russian).

[13] Lyubimova R.A. About one differential equation with invariant straight lines. Differential and integral equations, Gorky Universitet. 1984, 8, 66-69; 1997, 1, 19-22 (in Russian).

[14] Puţuntică V., Şubă A. The cubic differential system with six real invariant straight lines along two directions. Studia Universitatis. 2008, no 8(13), 5-16.

[15] Puţuntică V., Şubă A. The cubic differential system with six real invariant straight lines along three directions. Bulletin of ASM. Mathematics. 2009, no 2(60), 111-130.

[16] Repeşco V. Cubic systems with degenerate infinity and straight lines of total parallel multiplicity six. ROMAI J. 2013, 9 (1), 133-146.

[17] Romanovski V.G., Shafer D.S. The center and cyclicity problems: a computational algebra approach. Boston, Basel, Berlin: Birkhäuser, 2009. 
[18] Sadovskii, A. P. On conditions for a center and focus for nonlinear oscillation equations. Differentsial'nye Uravneniya. 1979, 15 (9), 1716-1719 (in Russian).

[19] Schlomiuk D. Elementary first integrals and algebraic invariant curves of differential equations. Expositiones Mathematicae. 1993, 11, 433-454.

[20] Sibirski K. The number of limit cycles in the neighborhood of a singular point. Diff. Uravneniya. 1965, 1 (1), 51-66 (in Russian).

[21] Şubă A. Solution of the center problem for cubic systems with a bundle of three invariant straight lines. Bulletin of ASM. Mathematics. 2003, no 1(41), 91-101.

[22] Şubă A. Center problem for cubic differential systems with the line at infinity of multiplicity four. Carpathian. J. Math. 2022, 38, no 1, 217-222.

[23] Şubă A., Cozma D. Solution of the problem of the center for cubic system with two homogeneous and one non-homogeneous invariant straight lines. Bulletin of ASM. Mathematics. 1999, no 1(29), 37-44.

[24] Şubă A., Cozma D. Solution of the problem of the centre for cubic system with three invariant straight lines two of which a parallel. Bulletin of ASM. Mathematics. 2001, no 2(36), 75-86.

[25] Şubă A. and Cozma D. Solution of the problem of the center for cubic differential system with three invariant straight lines in generic position. Qual. Theory of Dyn. Systems. 2005, 6, 45-58.

[26] Şubă A., Repeşco V. Configurations of invariant straight lines of cubic differential systems with degenerate infinity. Scientific Bulletin of Chernivtsi University, Series "Mathematics". 2012, 2 (2-3), 177-182.

[27] Şubă A., Repeşco V. Cubic systems with degenerate infinity and invariant straight lines of total parallel multiplicity five. Bul. Acad. Ştiinţe Repub. Mold., Mat. 2016, no. 3(82), 38-56.

[28] Şubă A., Repeşco V., Puţuntică V. Cubic systems with invariant affine straight lines of total parallel multiplicity seven. Electron. J. Diff. Equ. 2013, 2013 (274), 1-22. http://ejde.math.txstate.edu/

[29] Şubă A., Vacaraş O. Cubic differential systems with an invariant straight line of maximal multiplicity. Annals of the University of Craiova. Mathematics and Computer Science Series. 2015, 42 (2), $427-449$.

[30] Vacaraş O. Cubic differential systems with two affine real non-parallel invariant straight lines of maximal multiplicity. Bul. Acad. Ştiinţe Repub. Mold., Mat. 2015, no. 3(79), 79-101.

[31] Żołądek H. and Llibre J. The Poincaré center problem. Journal of Dynamical and Control Systems. 2008, 14 (4), 505-535.

Received 16.11.2021

Шубе О., Вакараш О. Задача про иентр для кубічних диферениіальних систем з лінією на нескінченності та афінною дійсною інваріантною прямою загальної кратності чотири // Буковинський матем. журнал - 2021. - Т.9, №2. - С. 35-52.

У роботі розглядається система двох диференціальних рівнянь із кубічними многочленами у правих частинах, для якої змінні та коефіцієнти набувають дійсних значень. Для цієї системи точка $(0,0)$ є критичним типом центра або фокуса. Актуальною є проблема розрізнення цих типів (проблема центра). Початок координат є центром тоді і тільки тоді, коли всі ляпуновські величини $L_{1}, L_{2}, \ldots, L_{n}, \ldots$ рівні нулю. У випадках, коли система має чотири (три) різні афінні прямі у працях О. Шубе і Д. Козьми показано, що точка $(0,0)$ є центр тоді і тільки тоді, коли анулюються перші дві (сім) ляпунівські величини. 
У цих роботах під час розгляду проблеми центру не враховувалася кратність лінії на нескінченності $\left(m\left(l_{\infty}\right)\right)$ та афінних інваріантних) прямих $\left(\left(m\left(l_{j}\right)\right)\right.$. У даній статті ця задача розв'язана за наявності дійсної афінної інваріантної прямої $l_{1}$, здійснено класифікацію систем, для яких $m\left(l_{\infty}\right)+m\left(l_{1}\right) \geq 4$, і доведено, що початок координат є центром лише за рівності нулю перших чотирьох ляпуновських величин. При доведенні використовуються методи інтегровності Дарбу, оборотності Желондека та узагальненої симетрії Черкаса. Наведено приклади, що вказують на суттєвість вимоги $L_{1}=L_{2}=L_{3}=L_{4}=0$.

Ключові слова і фрази: кубічна диференціальна система, проблема центру, інваріантна пряма. 